\title{
Detector Concept for Neutron Tomography in the 10-15 MeV Energy Range
}

Frank Dietrich and James Hall Lawrence Livermore National Laboratory P.O. Box 808 Livermore, CA 94550 $\cdots+2=0$

liar o 6396

\section{OSTI}

\section{April, 1996}

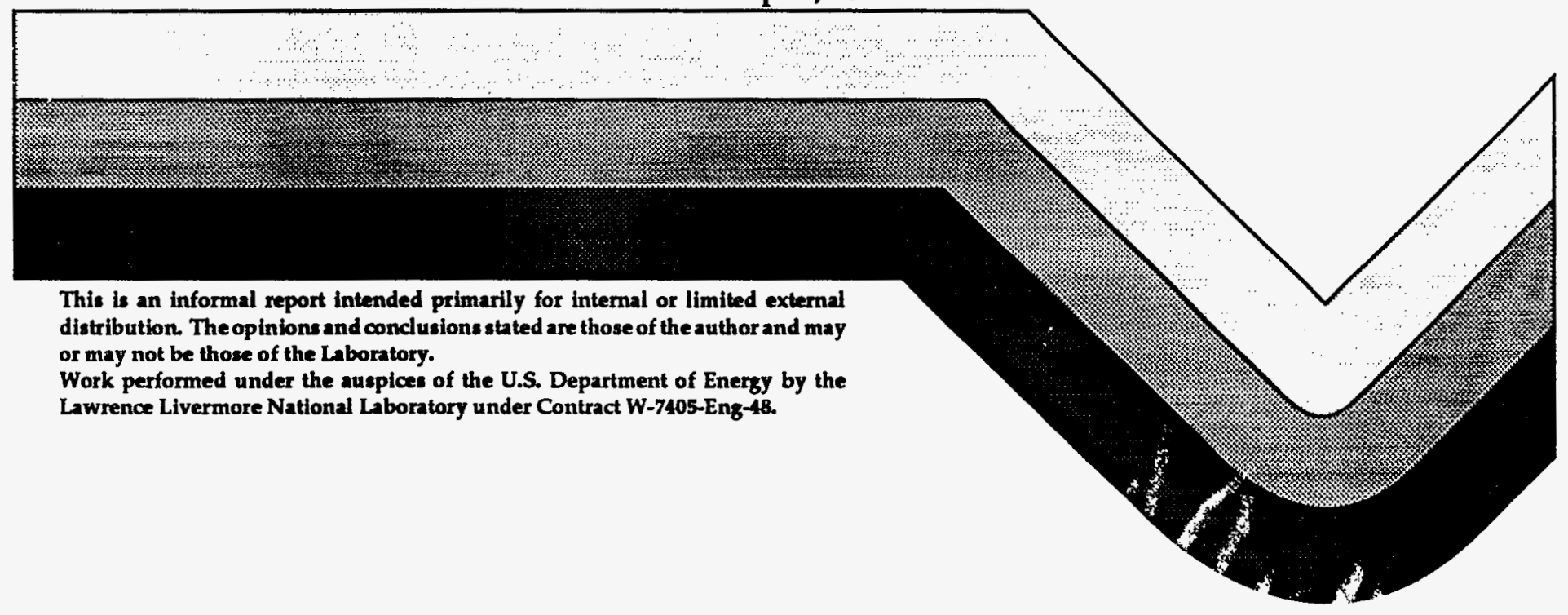




\section{DISCLAIMER}

This document was prepared as an account of work sponsored by an agency of the United States Government. Neither the United States Government nor the University of Califomia nor any of their employees, makes any warranty, express or implied, or assumes any legal liability or responsibility for the accuracy, completeness, or usefulness of any information, apparatus, product, or process disclosed, or represents that its use would not infringe privately owned rights. Reference herein to any specific commercial products, process, or service by trade name, trademark, manufocturer, or otherwise, does not necessarily constitute or imply its endorsement, recommendation, or favoring by the United States Government or the University of California. The views and opinions of authors expressed herein do not necessarily state or reflect those of the United States Government or the University of Californis, and shall not be used for advertising or product endorsement purposes.

This report has been reproduced

directly from the best available copy.

Available to DOE and DOE contractors from the Office of Scientific and Technical Information

P.O. Box 62, Oak Ridge, TN 37831

Prices available from (615) 576-8401, FTS 626-8401

Available to the public from the

National Technical Information Service

U.S. Department of Commerce

5285 Port Royal Rd.,

Springfield, VA 22161 


\title{
Detector Concept for Neutron Tomography in the 10 - $15 \mathrm{MeV}$ Energy Range
}

\author{
Frank Dietrich and James Hall \\ Lawrence Livermore National Laboratory
}

\begin{abstract}
Recent changes in the US nuclear weapons stockpile and its associated production complex have made nondestructive surveillance of weapons systems an increasingly important issue. In this paper we present the conceptual design for a neutron imaging system for use in the $10-15 \mathrm{MeV}$ energy range and discuss it potential applications in stockpile stewardship.
\end{abstract}

\section{Introduction}

Recent changes in the US nuclear stockpile and its associated production complex have made nondestructive surveillance of weapons systems an increasingly important issue. Historically, he integrity and/or reliability of stockpiled weapons systems has occasionally been called into question and the disassembly process required to re-certify the system has proven to be expensive and generally irreversible. The most common approach to nondestructive (and nonintrusive) inspection of complex sealed objects such as these is photon imaging (e.g. gamma radiography and tomography); however, while conventional photon imaging is an adequate tool for inspecting the condition of heavy metal (high-Z) parts within a device, it is generally not an effective means of determining whether voids, cracks or other significant structural defects exist in lighter (lowZ) materials within the device, particularly when those materials are shadowed by high-Z parts.

We have collaborated in recent experiments at the LANSCE /WNR facility which established the proofof-principle for radiography of thick targets using very high energy neutrons $(\approx 40-400 \mathrm{MeV})$ (Gavron, 1994). In those experiments (cf. Figure 1), a simple assembly consisting of a $2.54-\mathrm{cm}$-thick LiD disk sandwiched between two 5.08-cm-thick uranium slabs was used as a phantom target. Small holes $(4-12 \mathrm{~mm}$ ) drilled all or part of the way through the LiD were used to simulate defects. A position-sensitive multiwire detector with a thin tungsten converter plate was used to capture the neutron image with a resolution of $\approx 1 \mathrm{~mm}$. Because neutron radiography can be used to detect defects such as these in low- $Z$ materials even when those materials are heavily shielded (extremely difficult with photon radiography), this new technique has potential applications in stockpile surveillance.

In view of the fact that the neutron source requirements for some applications may be more easily met at much lower energies than those used in the WNR experiments, we are currently studying the feasibility of neutron doing radiography and tomography in the 10 $15 \mathrm{MeV}$ range. While the overall neutron transmission through thick targets is somewhat lower in this range than at higher energies (cf. Figure 2), this disadvantage is largely offset by the ability to use plastic-scintillator- based detectors which are significantly more efficient than those currently used for high-energy neutrons. The transmission contrast ratio in low- $\mathrm{Z}$ materials such as $\mathrm{LiD}$ is also more favorable at lower neutron energies thereby making it easier to detect defects. We have recently developed a conceptual design for a neutron imaging detector system for use in the low energy region that we believe will have reasonable efficiency $(\approx$ $30 \%$ ) and spatial resolution $(\approx 1 \mathrm{~mm})$.

\section{Computer Simulations}

We began our investigation of low-energy neutron probes by simulating the results of the WNR proof-ofprinciple experiment using 14-MeV neutrons with the LLNL-developed "COG" Monte Carlo radiation transport code. We have since also simulated a tomographic experiment and image reconstruction on a suitable object. The results of the COG simulation at $14 \mathrm{MeV}$ (cf. Figure 3) show that radiography with low energy (10 $15 \mathrm{MeV}$ ) neutrons should be capable of revealing features similar to those seen in the higher-energy WNR experiment. These results, along with our tomographic simulations of other phantom targets relevant to the weapons program, suggest that it should be possible to detect defects $\approx 1 \mathrm{~mm}$ in diameter in shielded, low- $Z$ materials during an exposure of several hours using a neutron generator of practical size (nominal output of $\approx$ $10^{12}$ neutrons per second). Optimizing the design of a full-scale tomographic imaging system capable of inspecting a weaponized nuclear device (including minimizing the required neutron source strength and mitigating the effects of multiple neutron scattering) will require a careful evaluation of radiation backgrounds and shielding requirements. We intend to study these design issues and evaluate potential tomographic reconstruction algorithms through a combination of detailed computer simulations and experiments at LLNL and other facilities.

\section{Conceptual Design}

Our current conceptual design for an imaging detector system for use in neutron tomography in the 10 $15 \mathrm{MeV}$ energy range is based on proven Nuclear Test Program technology (cf. Figure 4). We propose the use of a plastic scintillator fluor (e.g. BC-408) viewed by 
one or more high-efficiency, high-resolution CCD cameras. Our preliminary calculations indicate that a fluor of thickness $\approx 4 \mathrm{~cm}$ should have a detection efficiency in excess of $30 \%$ for $14 \mathrm{MeV}$ neutrons (this compares to an efficiency of $\approx 0.3 \%$ for the detector used in the higher-energy WNR experiment). The camera system would consist of a fast ( $f / 1.2)$ lens coupled to a backthinned, high-resolution ( $\approx 512 \times 512$ ) CCD imaging chip operating at liquid nitrogen temperatures to minimize thermal electronic noise. A thin metallic turning mirror would be used to reflect light from the scintillator fluor into the camera which would be placed in a shielded enclosure well out of the neutron beam path. (cf. Figure 5). Our calculations also indicate that the camera system should be capable of detecting the light generated by a single neutron interacting at the fluor; therefore an image intensifier should not be necessary unless we find that gating is required to mitigate scattered neutron backgrounds. The overall spatial resolution of the imaging system should be $\approx 1.50 \mathrm{~mm}$ at the fluor (this would correspond to a resolution of $\approx 0.75$ $\mathrm{mm}$ at the device location for the facility layout under consideration here).

\section{Technical Risk Assessment}

While continuing to play an active role in the highenergy neutron imaging experiments being carried out at LANL, we are working to refine and optimize the design of a low-energy $(10-15 \mathrm{MeV})$ neutron imaging system at LLNL. We are also working to improve our understanding of the technical risks associated with developing and using such a system for stockpile surveillance. We recently completed an experiment designed to measure scattered radiation backgrounds in a prototypical imaging system environment. This experiment was carried out in January of 1996 at the Ohio University Accelerator Laboratory (OUAL) in Athens, Ohio which features accelerator-driven DD and DT neutron sources and shielded detector caves similar to those envisioned in an actual neutron imaging facility. While the data from this experiment are still being analyzed, our initial impression is that scattering from sources external to the target (i.e. room scattering) can be satisfactorily controlled using well-designed collimators and shielding structures.

Two of the key technical risks which must be addressed during the development of a neutron imaging system involve the potentially adverse effects of prolonged exposure to high energy neutrons on materials usually found in nuclear devices, in particular the uranium and LiD components (integrated neutron fluxes are expected to be $\approx 10^{9} \mathrm{n} / \mathrm{cm}^{2}$ with our system). The radiation dose from induced radioactivity due to neutron activation of the device assembly must fall well below the intrinsic radiation level of the device itself within some reasonable time following the inspection process $(\approx 24$ hours). Detailed numerical simulations for one system currently in the stockpile suggest that neutron activation will not be a problem for the materials in the weapon's aeroshell or component support structures; however, our codes cannot account for activation of the uranium itself. This will be addressed separately in experiments planned for early FY97 at OUAL. In addition to the issue of neutron activation, we must also address the possible production of free hydrogen within $\mathrm{LiD}$ components in the device as a result of neutron bombardment. While we do not believe this will be a serious problem, we do plan to resolve the issue to the satisfaction of all concerned in experiments at OUAL. The results of these tests are essential to acceptance of this surveillance technique within the nuclear design community.

\section{References}

Gavron, A., "High Energy Neutron Radiography Observing the LiD," P-23 (LANL) memorandum written on behalf of A. Bridge, C. Morris, M. Murray, S. Seestrom, J. Ulimann, W. Abfalterer, and A. Gavron of LANL and F. Dietrich and T. Wenaus of LLNL (December 7, 1994).
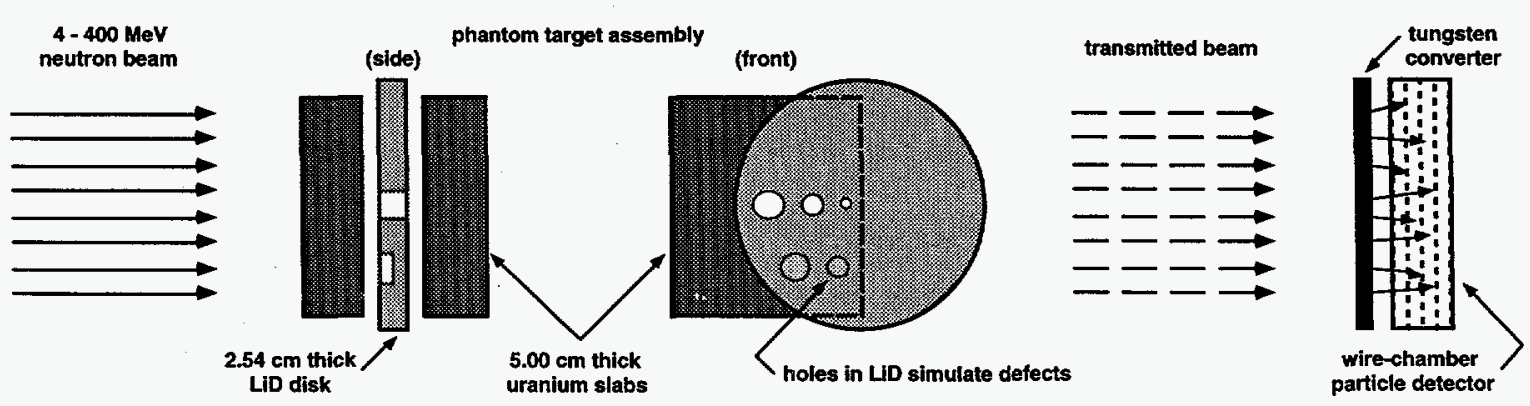

Figure 1: Recent experiments carried out at the LANSCE/WNR facility at LANL have demonstrated the potential for using fast neutron radiography for stockpile stewardship. Neutrons with energies of up to $\approx 400 \mathrm{MeV}$ were used to irradiate a uranium and $\mathrm{LiD}$ phantom target assembly. Small holes $(4-12 \mathrm{~mm})$ drilled all or part of the way through the LiD were used to simulate defects. A position sensitive multiwire detector (resolution $\approx 1 \mathrm{~mm}$ ) was used to record the image. 


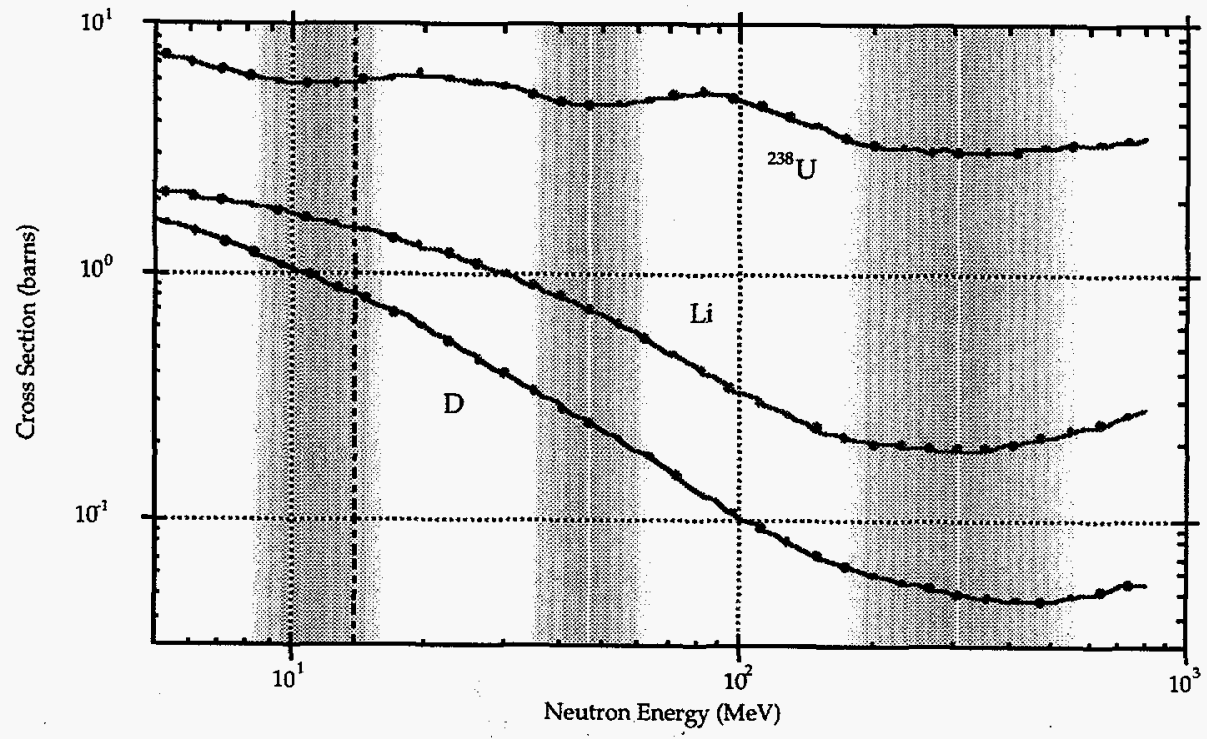

Figure 2: The total neutron interaction cross sections shown here were obtained from A. Gavron (LANL). Dips in the uranium cross section determine energy "windows" suitable for neutron imaging (i.e., regions of relatively high transmission). The higher the lithium and deuterium cross sections are in these regions, the higher the transmission contrast ratio is, thus making it easier to detect defects.

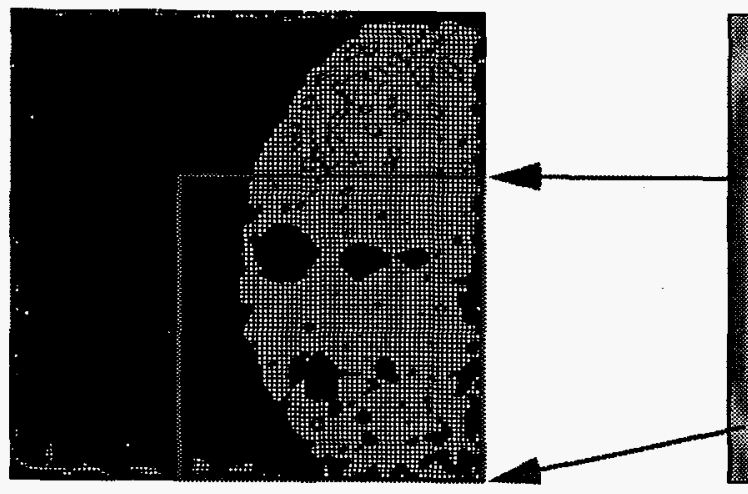

LANSCE/WNR image
$(\approx 50-400 \mathrm{MeV}$ neutrons)

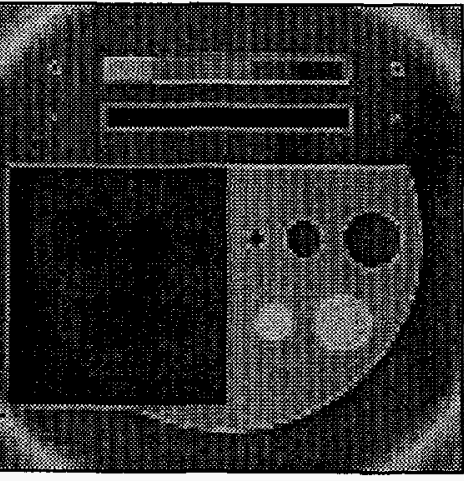

COG simulation (14 MeV neutrons)

Figure 3: This figure shows the experimental image of the LANSCE/WNR phantom target taken using $\approx 50-400$ $\mathrm{MeV}$ neutrons compared to the COG Monte Carlo simulation of a similar phantom for the case of $14 \mathrm{MeV}$ neutrons. Line-outs taken through corresponding regions in the images show similar contrast ratios in the vicinity of the LiD defects in spite of the large difference in incident neutron energies. 


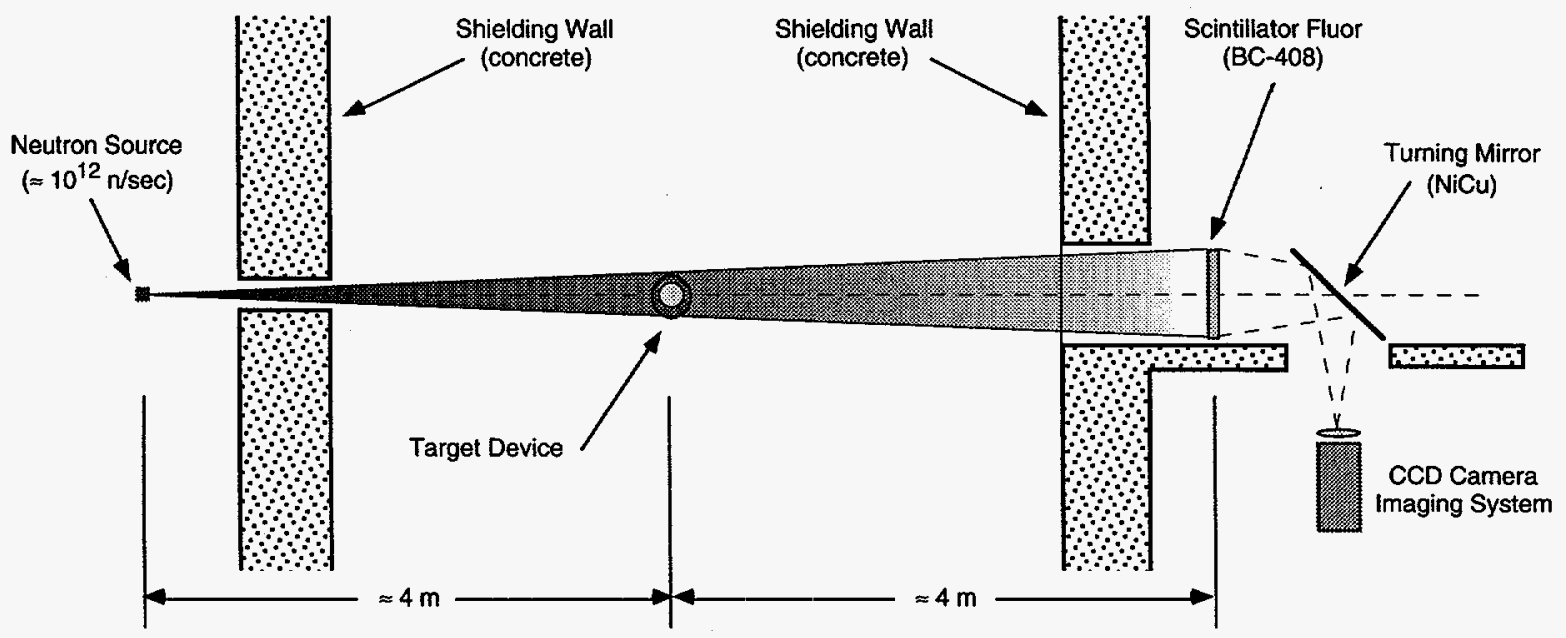

Figure 4: We are currently developing the conceptual design for an imaging system for use in neutron radiography and tomography at low energies $(10-30 \mathrm{MeV})$ using proven Nuclear Test Program technology. The plastic scintillator fluor coupled with one or more high-resolution CCD cameras should provide both adequate sensitivity and position resolution at the target.

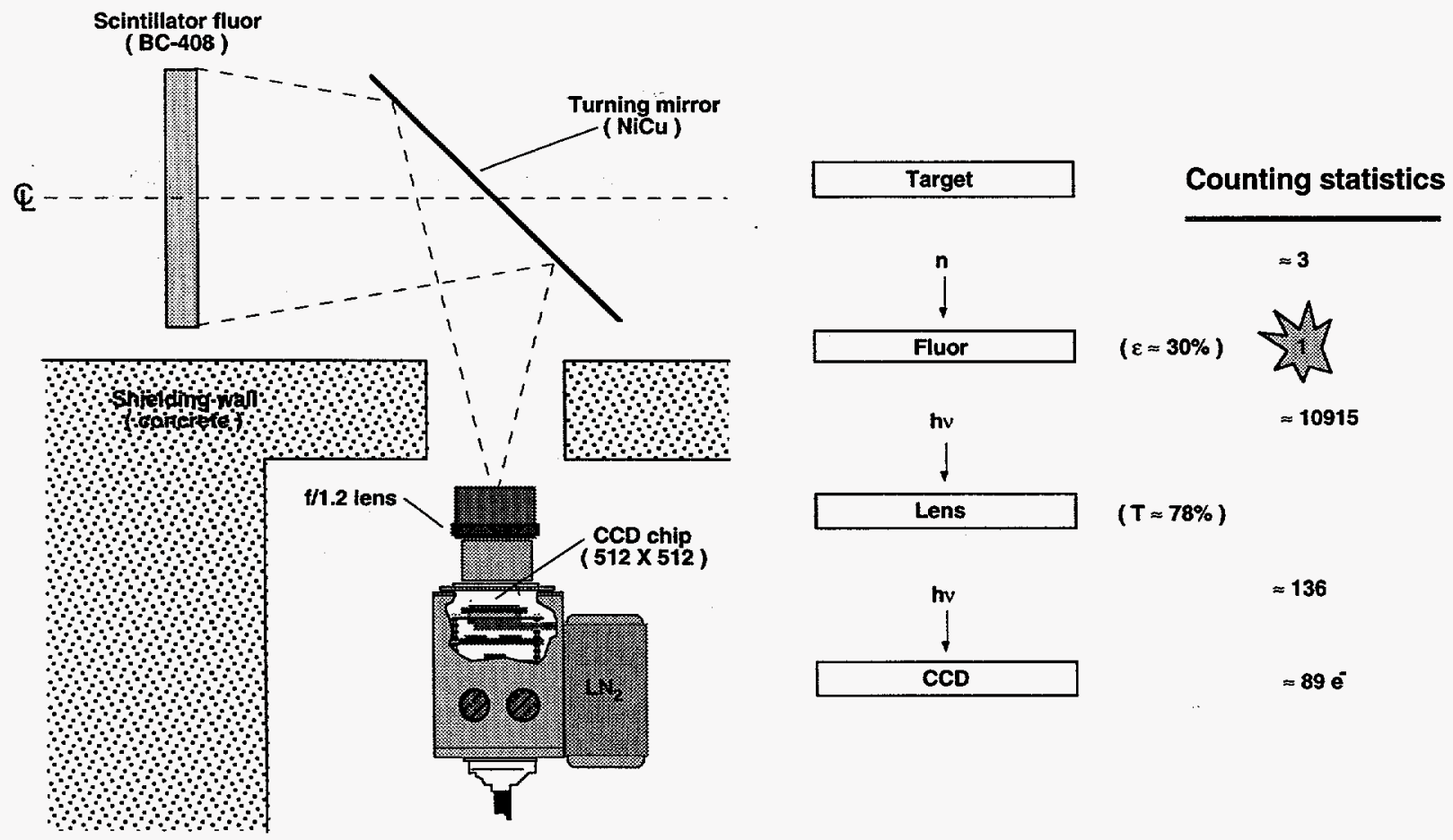

Figure 5: The camera system would consist of a fast $(f / 1.2)$ lens coupled to a back-thinned, high-resolution $(\approx 512$ $X$ 512) CCD imaging chip operating at liquid nitrogen temperatures to minimize thermal electronic noise. A thin metallic turning mirror would be used to reflect light from the scintillator fluor into the camera which would be placed in a shielded enclosure well out of the neutron beam path. 
,

. 